Swarthmore College

Works

8-1983

\title{
APL And The Numerical Solution Of High-Order Linear Differential Equations
}

\author{
N. A. Gershenfeld \\ Edward H. Schadler , '81 \\ Oleksa-Myron Bilaniuk \\ Swarthmore College
}

Follow this and additional works at: https://works.swarthmore.edu/fac-physics

Part of the Physics Commons

Let us know how access to these works benefits you

\section{Recommended Citation}

N. A. Gershenfeld; Edward H. Schadler , '81; and Oleksa-Myron Bilaniuk. (1983). "APL And The Numerical Solution Of High-Order Linear Differential Equations". American Journal Of Physics. Volume 51, Issue 8. 743-746. DOI: 10.1119/1.13158

https://works.swarthmore.edu/fac-physics/351

This work is brought to you for free by Swarthmore College Libraries' Works. It has been accepted for inclusion in Physics \& Astronomy Faculty Works by an authorized administrator of Works. For more information, please contact myworks@swarthmore.edu. 


$$
0 \leqslant \sum_{\substack{\beta \\ \beta \neq \alpha}}^{\dot{S}}-L_{\beta \alpha} \leqslant L_{\alpha \alpha}-1 .
$$

For systems in thermodynamic equilibrium, the off-diagonal matrix elements of the stability matrix $\left[U_{\alpha \beta}\right]$ are not constrained in sign. For very many thermodynamic systems, the matrix elements $U_{\alpha \beta}$ and $U^{\alpha \beta}(\alpha \neq \beta)$ do have opposite signs. The second law of thermodynamics requires the inequalities $(\mathrm{A} 2)$ but not the inequalities (A3). If inequalities of the form (A3) or (A5) were found to be universally satisfied by systems in thermodynamic equilibrium with respect to the natural thermodynamic variables, our understanding of the second law of thermodynamics would have to be sharpened.

${ }^{1}$ P. Samuelson, in The Collected Scientific Papers of Paul A. Samuelson, Vol. I, edited by J. E. Stiglitz (MIT, Cambridge, 1966), p. 639.
${ }^{2}$ E. Fermi, Thermodynamics (Dover, New York, 1937), p. 111.

${ }^{3}$ (a) F. Reif, Fundamentals of Statistical and Thermal Physics (McGrawHill, New York, 1965), p. 298. (b) J. E. Mayer and M. G. Meyer, Statistical Mechanics, 2nd ed. (Wiley, New York, 1977), p. 108. (c) P. S. Epstein, Textbook of Thermodynamics (Wiley, New York, 1937), p. 379. (d) H. B. Callen, Thermodynamics (Wiley, New York, 1960), p. 135.

${ }^{4}$ P. S. Epstein, Textbook of Thermodynamics (Wiley, New York, 1937), p. 382 (Le Châtelier-Braun principle).

${ }^{5}$ (a) H. B. Callen, Thermodynamics (Wiley, New York, 1960), p. 139 (Le Châtelier-Braun principle). (b) L. D. Landau and E. M. Lifshitz, Statistical Physics (Pergamon, London, 1958), p. 63. (c) I. P. Bazarov, Thermodynamics (Pergamon, New York, 1964), p. 271 (Le Châtelier-Braun principle).

${ }^{6}$ J. DeHeer, J. Chem. Educ. 34, 375 (1957).

${ }^{7}$ P. Ehrenfest, J. Russ. Phys. Soc. 41, 347 (1909), Z. Phys. Chem. 77, 227 (1911).

${ }^{8}$ R. Gilmore, Catastrophe Theory for Scientists and Engineers (Wiley, New York, 1981), p. 224 et seq.

${ }^{9}$ R. Gilmore, J. Chem. Phys. 76, 5551 (1982).

${ }^{10}$ F. Weinhold, J. Chem. Phys. 63, 2479, 2484, 2488, 2496 (1975).

\title{
APL and the numerical solution of high-order linear differential equations
}

\author{
Neil A. Gershenfeld \\ Bell Laboratories, Murray Hill, New Jersey 07974
}

Edward H. Schadlera) and O. M: Bilaniuk

Department of Physics, Swarthmore College, Swarthmore, Pennsylvania 19081

(Received 12 April 1982; accepted for publication 18 September 1982)

\begin{abstract}
An $N$ th-order linear ordinary differential equation is rewritten as a first-order equation in an $N \times N$ matrix. Taking advantage of the matrix manipulation strength of the APL language this equation is then solved directly, yielding a great simplification over the standard procedure of solving $N$ coupled first-order scalar equations. This eases programming and results in a more intuitive algorithm. Example applications of a program using the technique are given from quantum mechanics and control theory.
\end{abstract}

\section{INTRODUCTION}

A task of a physics student, and that of a physicist in general, frequently leads to a differential equation. Some intuition about the nature of its solution is invariably very helpful. Working interactively with a computer that generates solutions and provides graphical output is an excellent way to devêlop such intuition. This note reports on a great simplification possible for high-order linear differential equations taking advantage of the matrix manipulation capabilities of the APL computer language. The matrix manipulation flexibility of APL allows an $N$ th-order linear ordinary differential equation to be solved numerically in terms of a single first-order equation in an $N \times N$ matrix, instead of the standard system of $N$ first-order scalar equations. This greatly simplifies programming and results in more intuitively understandable algorithm. A program implementing this technique is described below, and exam- ples are given from quantum mechanics and control theory.

\section{MATRIX FORMULATION}

The general form of the problem is

$$
A_{N}(t) \frac{d^{N} z}{d t^{N}}+\cdots+A_{1}(t) \frac{d z}{d t}+A_{0}(t) z=F(t)
$$

with initial conditions

$$
\left.\frac{d^{N-1 \dot{z}}}{d t^{N-1}}\right|_{t=t_{0}}=K_{N-1}, \ldots,\left.\frac{d z}{d t}\right|_{t=t_{0}}=K_{1}, z\left(t_{0}\right)=K_{0} \text {. }
$$

The standard method for numerically solving such an $N$ th-order initial-value problem entails converting it ot the system of $N$ first-order equations 


$$
\begin{aligned}
z_{0}= & z, \\
z_{1}= & \frac{d z_{0}}{d t}, \\
\vdots & \\
z_{N-1}= & \frac{d z_{N-2}}{d t}, \\
z_{N}= & \frac{1}{A_{N}}\left[F(t)-A_{N-1}(t) z_{N-1}-\cdots\right. \\
& \left.-A_{1}(t) z_{1}-A_{0}(t) z_{0}\right],
\end{aligned}
$$

and solving this system subject to the appropriate initial values. However, and this is the crux of the present approach, an $N$ th-order differential equation may also be written as a single first-order equation in terms of an $N$ vector $\mathbf{V}$ :

$$
\frac{d}{d t} \mathbf{V}=M \mathbf{V}+\mathbf{A} \text {, }
$$

where $M$ is the companion matrix

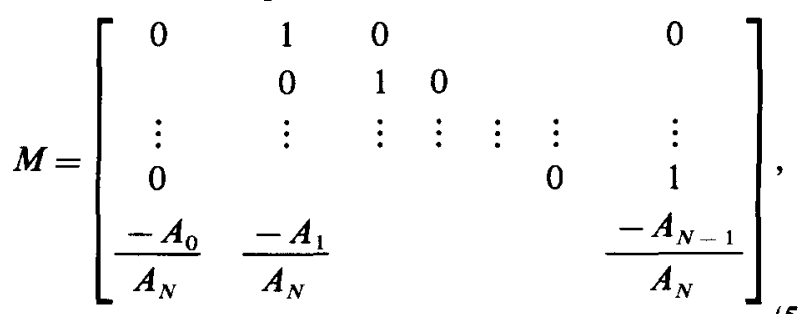

$\mathrm{V}$ is the column vector

$$
\mathbf{V}=\left[\begin{array}{c}
z \\
d z / d t \\
\vdots \\
\frac{d^{N-1} z}{d t^{N-1}}
\end{array}\right],
$$

and $\mathbf{A}$ is the column vector containing the "driving force" $F(t)$ :

$$
\mathbf{A}=\left[\begin{array}{c}
0 \\
\vdots \\
F / A_{N}
\end{array}\right]
$$

$V(0)$ specifies the initial conditions. This idea is attributable to $\mathrm{H}$. Wronski (1778-1853). ${ }^{1}$

Because APL manipulates matrices as readily as scalars, this matrix prolem may be treated as any first-order differential equation and solved accordingly. Herein lies the simplification that makes the programming so straightforward.

\section{NUMERICAL SOLUTION}

There is a great variety of numerical algorithms available for solving first-order differential equations; the method we chose is a fourth order Adams-Bashforth (AB) predictor with an Adams-Moulton (AM) corrector with variable step size. ${ }^{2}$ The first-order problem to be solved now is

$$
\frac{d z}{d t}=F(z, t), \quad z\left(t_{0}\right)=z_{0} .
$$

Writing $z\left(t_{i}\right)$ as $z_{i}$, the predictor generates a value for $z_{i+1}$ given $z_{i}, \ldots, z_{i-3}$ by

$$
\begin{aligned}
z_{i+1}^{p}= & z_{i}+\frac{\Delta t}{24}\left[55 F\left(z_{i}, t\right)-59 F\left(z_{i-1}, t_{i-1}\right)\right. \\
& \left.+37 F\left(z_{i-2}, t_{i-2}\right)-9 F\left(z_{i-3}, t_{i-3}\right)\right] .
\end{aligned}
$$

This value is then improved with the corrector

$$
\begin{aligned}
z_{i+1}^{c}= & z_{i}+\frac{\Delta t}{24}\left[9 F\left(z_{i+1}, t_{i+1}\right)+19 F\left(z_{i}, t_{i}\right)\right. \\
& \left.-5 F\left(z_{i-1}, t_{i-1}\right)+F\left(z_{i-2}, t_{i-2}\right)\right] .
\end{aligned}
$$

The difference between the predicted and corrected values gives a measure of the local error:

$$
\delta \approx \frac{1}{10}\left|z_{i+1}^{c}-z_{i+1}^{p}\right| .
$$

If this error falls outside the desired bounds the step size can be readjusted. ${ }^{3}$

A simple method is to correct with the factor

$$
q=\left(\frac{\epsilon}{2 \delta}\right)^{1 / 5} \text {, }
$$

where $\epsilon$ is the desired local error. If the error is too small $(\delta<0.1 \epsilon)$, the step is replaced with $q \Delta t$ (or $4 \Delta t$ if $q>4$ ). Similarly, if $\delta>\epsilon$ the step is replaced with $q \Delta t$ (or $0.1 \Delta t$ if $q<0.1$ ). There are many subtleties in choosing a method for step-size control; the simple criterion used here was chosen in keeping with the intended educational use of the algorithm. ${ }^{4}$

The method requires four equally spaced points to generate a new one. At the start of the calculation, or when the step size is changed, these four points must be generated by a "starter" method that does not require previous points. A fourth-order Runge-Kutta (RK) routine ${ }^{5}$ was used as the starter. It is given by

$$
\begin{aligned}
& K_{1}=\Delta t F\left(z_{i}, t_{i}\right), \\
& K_{2}=\Delta t F\left(z_{i}+K_{1} / 2, t_{i}+\Delta t / 2\right), \\
& K_{3}=\Delta t F\left(z_{i}+K_{2} / 2, t_{i}+\Delta t / 2\right), \\
& K_{4}=\Delta t F\left(z_{i}+K_{3}, t_{i}+\Delta t\right), \\
& z_{i+1}=z_{i}+\frac{1}{6}\left(K_{1}+2 K_{2}+2 K_{3}+K_{4}\right) .
\end{aligned}
$$

The RK routine, once started, could be used for the rest of the calculation. However, RK is less efficient, requiring four evaluations of the function $F$ to AB-AM's two. Moreover, step-size control is quite difficult with the RK routine. By comparison, the fourth-order AB-AM predictorcorrector set provides a good balance between accuracy and complexity for most physical problems. Although some additional work is required to provide the needed starting points, the increased accuracy justifies the effort. The ease with which automatic step-size control may be achieved is an added bonus.

A word of caution should be added about the use of the algorithm. It can be shown ${ }^{6}$ that any simple linear technique, such as the $\mathrm{AB}-\mathrm{AM}$ set, can be unstable, with the error increasing without bound during a calculation. In particular, for a stiff problem (a stiff problem can be loosely defined as one having widely varying time scales ${ }^{7}$ ), the method may quickly fail. There are stable techniques for stiff problems; these typically require the solution of an implicit equation or the calculation of a Jacobian. ${ }^{8}$ The AB-AM method was chosen for its understandability, simplicity, and acceptably tame behavior for reasonable problems (such as the examples to follow), however, it is possible for the method to fail in a noncatastrophic and hence non- 
obvious fashion. Therefore, with this as with any other numerical technique, one should check that the answers obtained are physically plausible.

\section{APPLICATIONS}

Figures 1 and 2 show two examples of the instructional possibilities of the program. Figure 1 gives the radial probability density $P_{n l}(r)=r^{2}\left[R_{n l}(r)\right]^{2}$ for the electron in a hydrogen atom for the $l=0$ states $n=1,2,3,4$. The associated Laguerre polynomials, $L_{q}^{j}(\rho)$, which enter into the radial function

$R_{n l}(r)=-\left[\left(\frac{2 Z}{n a_{0}}\right)^{3} \frac{(n-l-1)}{2 n[(n+l) !]^{3}}\right]^{1 / 2} e^{-\rho / 2} \rho^{l} L_{n+l}^{2 l+1}(\rho)$,

where $\rho=2 Z r / n a_{0}$, were generated from Laguerre's differential equation

$$
\left(\rho \frac{d^{2}}{d \rho^{2}}+(j+1-\rho) \frac{d}{d \rho}+(q-j)\right) L_{q}^{j}(\rho)=0 .
$$

The equivalent matrix formulation for this problem is

$$
\begin{aligned}
\frac{d}{d \rho}\left[\begin{array}{c}
L \\
\frac{d L}{d \rho}
\end{array}\right] \\
\quad=\left[\begin{array}{cc}
0 & 1 \\
-\frac{(q-j)}{\rho}-\frac{(j+1-\rho)}{\rho}
\end{array}\right]\left[\begin{array}{c}
L \\
\frac{d L}{d \rho}
\end{array}\right]+\left[\begin{array}{l}
0 \\
0
\end{array}\right] .
\end{aligned}
$$

The answers were generated with a local error of $10^{-6}$, giving an absolute error on the order of $0.1 \%$.

The simple manner in which the coefficients containing $Z, n$, and $l$ enter into this algorithm permits the student to explore the states of hydrogenic atoms that are not canonical textbook examples.

Figure 2 gives the solution of the classic control theory problem of balancing a rod by moving a platform that supports the rod; this is very similar to the problem of stablizing a rocket at launch. In the present example a rod of length $l$ and mass $m$ is hinged on a movable base of mass $M$. The angle $\theta$ measures the inclination of the rod from verti-

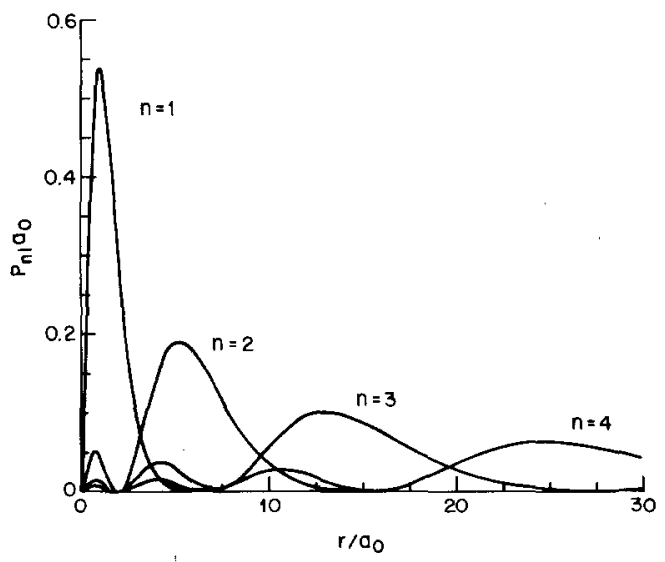

Fig. 1. Computed solutions for the probability density $\boldsymbol{P}_{n l}(r)=r^{2}\left[R_{n i}(r)\right]^{2}$ for an $s$ electron in a hydrogen atom with $n=1,2,3,4$; the axes are normalized by the Bohr radius $a_{0}$. The APL matrix stratagem was used to compute the $R_{n l}$ from Laguerre's differential equation. The area under each curve has been verified to be equal to one to within 0.0001 .

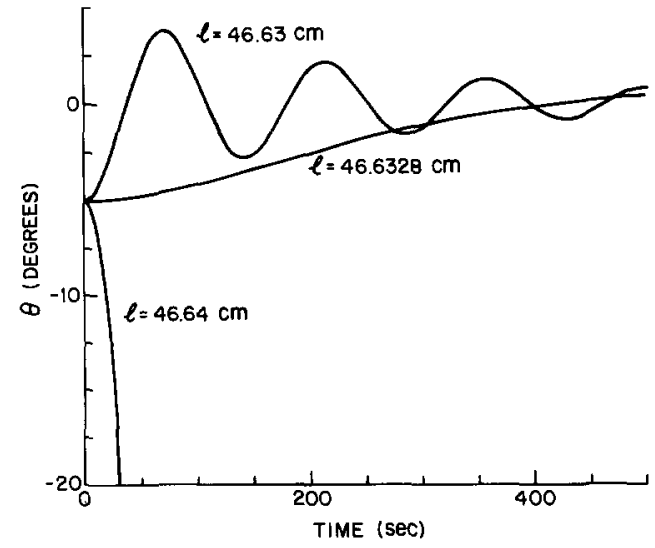

Fig. 2. Stable, critical, and unstable solutions for a simple model of rocket control at launch. A rod is hinged at its base and mounted on a platform which moves horizontally in response to a force that is proportional to the angle of the rod from vertical and its derivative. In the example chosen, a steel rod with a radius of $1 \mathrm{~cm}$ and length as indicated in the figure hinges on a base of $500 \mathrm{~g}$. The applied force is $F=500[5000 \theta+\dot{\theta}]$, where $\theta$ is the angle of the rod from vertical. The masses corresponding to the lengths $l_{1}=46.63, l_{2}=46.6328$, and $l_{3}=46.64 \mathrm{~cm}$ are $m_{1}=2050.90$, $m_{2}=2051.02$, and $m_{3}=2051.34 \mathrm{~g}$. The initial condition for each case is a start from rest at $-5 \mathrm{deg}$

cal. A horizontal correction force, proportional to $\theta$ and its derivative, $F=M(a \theta+b \dot{\theta})$, is applied to the base. For small $\theta$ the system's behavior is given by ${ }^{10}$

$$
\ddot{\theta}+\frac{3 b}{(\mu+4) l} \dot{\theta}+\frac{3[a-(1+\mu) g]}{(\mu+4) l} \theta=0,
$$

with $\mu=m / M$. The matrix problem to be solved is

$$
\begin{aligned}
\frac{d}{d \theta}\left[\begin{array}{l}
\theta \\
\dot{\theta}
\end{array}\right]= & {\left[-\frac{3[a-(1+\mu) g]}{(\mu+4) l}-\frac{3 b}{(\mu+4) l}\right]\left[\begin{array}{c}
\theta \\
\dot{\theta}
\end{array}\right] } \\
& +\left[\begin{array}{l}
0 \\
0
\end{array}\right] .
\end{aligned}
$$

For the system to recover from small displacements the coefficients must be positive, which implies $b>0$ and $a>(1+\mu) g$. The curves given are for a steel rod with a radius of $1 \mathrm{~cm}, M=500 \mathrm{~g}, a=5000$, and $b=1$; this gives a critical length of $46.6328 \mathrm{~cm}$. The salient features of this system, which may not be obvious from the differential equation, clearly emerge when the length is varied about the critical value. A rod of critical length smoothly recovers from a displacement, while a longer length quickly loses stability and a shorter length oscillates about equilibrium. The sharpness of this transition and the existence of a narrow region of acceptable length has obvious implications for the design or analysis of a similar system.

\section{CONCLUSION}

The above two cases are representative of a great number of applications. For example, the behavior of a damped harmonic oscillator comes acrośs more clearly when one can vary all parameters at will and thereby see how the system responds under conditions not readily accessible in the laboratory. A more formal analytic solution ${ }^{11}$ can frequently be bypassed while still acquiring a full physical understanding of the system. Another area of use is the physics covered by Bessel's equation, which becomes more 
explicit after the intuition building experience of watching the solutions develop for a variety of conditions.

The technique described in this note has limitations; use outside of a reasonable nonstiff problem may result in an obvious or hidden error. Acknowledging these restrictions, however, the use of the APL matrix stratagem described in this note has made high-order linear differential equations much more tractable for the authors; it is our hope that the reader will find the approach equally rewarding. A copy of the APL program using this algorithm along with a fuller description of the method may be obtained by writing to $O$. M. Bilaniuk, Department of Physics, Swarthmore College, Swarthmore, PA 19081. An excellent statement of the computational philosophy which underlies this work may be found in Ref. 12.

\section{ACKNOWLEDGMENTS}

We express our thanks to David Clemens for the graphics package, to David Rosen for his mathematical advice, and to Allen Blaer for his inspiration and assistance. a) Present address: 110 Woodhaven Drive, Scotia, NY 12302.

'E. L. Ince, Ordinary Differential Equations (Dover, New York, 1956), p. 116.

${ }^{2}$ R. Beckett and J. Hurt, Numerical Calculations and Algorithms (McGraw-Hill, New York, 1967), pp. 198-199.

${ }^{3}$ R. L. Burden, J. D. Faives, and A. C. Reynolds, Numerical Analysis (Prindle, Weber and Schmidt, Boston, 1978), p. 268.

${ }^{4}$ L. F. Shampine and M. K. Gordon, Computer Solution of Ordinary Differential Equations: The Initial Value Problem (Freeman, San Francisco, 1975), p. 109.

${ }^{5} \mathrm{C}$. W. Gear, Numerical Initial Value Problems in Ordinary Differential Equation (Prentice-Hall, Englewood Cliffs, NJ, 1971), p. 35.

${ }^{6}$ Shampine and Gordon, Ref. 4, p. 126

'Gear, Ref. 5, p. 209.

${ }^{8}$ Gear, Ref. 5, pp. $212-226$.

${ }^{9}$ M. A. Morrison, T. L. Estle, and N. F. Land, Quantum States of Atoms, Molecules, and Solids (Prentice-Hall, Englewood Cliffs, NJ, 1976), p. 57.

${ }^{10} \mathrm{~K}$. Ogata, Modern Control Engineering (Prentice-Hall, Englewood Cliffs, NJ, 1970), p. 278.

${ }^{11}$ A. Ricchinto and A. Tozzi, Am. J. Phys. 50, 176 (1982).

${ }^{12}$ F. Acton, Numerical Methods that Work (Harper and Row, New York, 1970), pp. 129-156.

\title{
Solar escape
}

\author{
A. Z. Hendel \\ Department of Physics, University of Michigan, Ann Arbor, Michigan 48109
}

(Received 30 June 1980; accepted for publication 16 September 1982)

If we try to calculate the minimum velocity a spaceship needs to escape from the solar gravitational field we are likely to get a wrong answer. We illustrate the difficulties with several examples and we give a simple method to solve a certain class of problems.

\section{INTRODUCTION}

There is an elementary physics problem to which we are likely to get the wrong answer:

What minimum velocity with respect to the Earth does a spaceship need near the surface of the Earth to escape from the solar gravitational field, neglecting air resistance and the spin of the Earth but not its orbital motion?

We invite the readers to solve this problem and to compare their answers with the ones we will give in this paper. Otherwise, the paper will appear to be trivial.

We found that almost everybody has difficulties with this kind of problem. All eight textbooks in which we found similar problems have wrong answers. ${ }^{1}$ In Sec. II below, we will point out what the difficulties are and how to avoid them, and we will recommend some generally useful procedures.

In Sec. III, we will solve this problem, using the best frames of reference, list correct and incorrect solutions, and point out common mistakes.

In Sec. IV, we will do the problem entirely in the solar frame to show explicitly the difficulties that arise when the best frame is not used.
In Sec. V, we list additional problems with similar difficulties.

\section{NON-NEGLIGIBLE ENERGY}

In an elastic two-particle interaction, the energy exchanged is zero only in the center-of-mass frame. If one of the particles has an infinite mass its energy change is zero only in the reference frame of the infinitely massive particle. In any other reference frame even an infinitely massive body absorbs (positive or negative) energy. This is obvious in cases such as that of a ball that rebounds from the top of an upward moving elevator in a perfectly elastic collision, where the energy gained by the ball is lost by the elevator and this energy is negligible with respect to that of the elevator but it can be huge with respect to that of the ball. Also, in a "slingshot" trajectory in the solar frame, a spaceship gains energy in the vicinity of Jupiter, energy that is lost by Jupiter. Failure to consider the second body and its energy can lead to mistakes. In most textbook problems, this failure does not matter since we choose the one ("best") reference frame (usually that of the Earth) in which the second body does not need to be taken into account. We 